\title{
A useful correspondence principle in the theory of linear materials
}

\author{
KUMBAKONAM R. RAJAGOPAL and ALAN WINEMAN
}

Department of Applied Mechanics and Engineering Science, The University of Michigan, Ann Arbor, Michigan 48109, USA

(Received May 23, 1979)

\section{Introduction}

The assumption that the stress at a particle is a linear functional of the infinitesimal strain history at the particle is usually expressed in the following form:

$$
\boldsymbol{\sigma}(t)=\boldsymbol{L}\left[\left.\boldsymbol{\varepsilon}(s)\right|_{-\infty} ^{t}\right] .
$$

Specific representations for $\boldsymbol{L}$ lead to well known models for material behavior. For example, the representations appropriate to linear elasticity and non-aging viscoelasticity [1], respectively, are

$$
\boldsymbol{L}\left[\left.\boldsymbol{\varepsilon}(s)\right|_{-\infty} ^{\boldsymbol{t}}\right]=\boldsymbol{E} \boldsymbol{\varepsilon}(t)
$$

and

$$
\boldsymbol{L}\left[\left.\boldsymbol{\varepsilon}(s)\right|_{-\infty} ^{\mathfrak{t}}\right]=\boldsymbol{G}(0) \boldsymbol{\varepsilon}(t)+\int_{0}^{t} \boldsymbol{\varepsilon}(t-s) d \boldsymbol{G}(s),
$$

where $\boldsymbol{E}$ is a constant and $\boldsymbol{G}(s)$ is a tensor-valued function of bounded variation on every finite subinterval.

Our purpose in this note is to point out that many results which have been derived separately in linear elasticity or viscoelasticity, for both aging and non-aging materials, depend only on the linearity of the response functional and not on the properties of a specific representation. To emphasize this point, we derive a tensorial property and analyze two typical boundary value problems.

\section{A tensorial property, material symmetry restrictions}

Let $X$ denote a Cartesian reference frame. Let $\sigma_{i j}^{X}(s)$ and $\varepsilon_{k l}^{X}(s), s \in(-\infty, t]$, denote the components of stress and strain tensorial histories at a particle with respect to $X$. We define a linear material to be one for which the constitutive equation with 
respect to each Cartesian reference frame $X$ is

$$
\sigma_{i j}^{X}(t)=\mathscr{F}_{i j k k}^{X}\left[\left.\varepsilon_{k l}^{X}(s)\right|_{-\infty} ^{t}\right] \text {, }
$$

where $\mathscr{F}_{i j k l}^{X}$ is a linear functional for each choice of the indices $i, j, k$, and $l$. From the symmetry properties of $\sigma_{i j}^{X}$ and $\varepsilon_{k l}^{X}$ it follows that

$$
\mathscr{F}_{i j k l}^{\mathrm{X}}=\mathscr{F}_{j i k l}^{\mathrm{X}}=\mathscr{F}_{i j l k}^{\mathrm{X}} \text {. }
$$

In a second Cartesian reference frame $X^{\prime}$, the constitutive equation is written as

$$
\sigma_{m n}^{X^{\prime}}=\mathscr{F}_{\text {mnpq }}^{X^{\prime}}\left[\left.\varepsilon_{\text {pq }}^{X^{\prime}}(s)\right|_{-\infty} ^{t}\right] \text {, }
$$

where $\mathscr{F}_{\text {mnpq }}^{\mathrm{X}^{\prime}}$ denotes another set of linear functionals. These functionals satisfy symmetry properties analogous to those in equation (5). We first show that $\mathscr{F}_{i j k l}^{\times}$are components with respect to $X$ of a fourth-order tensor-valued functional. This result was derived in [2]. A modified derivation is presented here along with some extensions of this result.

Let $\boldsymbol{A}$ denote the orthogonal transformation which relates frames $X$ and $X^{\prime}$. Since $\boldsymbol{A}$ is time independent, it can be represented through a set of constants $A_{i j}$ such that

$$
A_{i j} A_{i k}=A_{j i} A_{k i}=\delta_{j k} \text {. }
$$

For each $t$ and $s \in(-\infty, t]$, the stress and strain histories are related by

$$
\sigma_{i j}^{\mathrm{X}}(t)=A_{m i} A_{n j} \sigma_{m n}^{\mathrm{X}^{\prime}}(t), \quad \varepsilon_{\mathrm{pq}}^{\mathrm{X}^{\prime}}(s)=A_{\mathrm{p} k} A_{q l} \varepsilon_{k l}^{\mathrm{X}}(s) \text {. }
$$

Combining equations (4), (6), and (8), using the linearity of the functionals and the fact that $A_{i j}$ are constants, one obtains

$$
\mathscr{F}_{i j k l}^{\mathrm{X}}\left[\left.\varepsilon_{k l}^{\mathrm{X}}(s)\right|_{-\infty} ^{t}\right]=A_{m i} A_{n j} A_{p k} A_{\mathrm{ql}} \mathscr{F}_{m n p q}^{\mathrm{X}^{\prime}}\left[\left.\varepsilon_{k l}^{\mathrm{X}}(s)\right|_{-\infty} ^{t}\right] \text {. }
$$

This result must hold for all strain histories. Now, define the scalar null history $O(s)=0, s \in(-\infty, t]$. Let $\bar{k}$ and $\bar{l}$ denote fixed choices of indices $k$ and $l$. Consider strain histories of the following form:

$$
\varepsilon_{k l}^{X}(s)=\left\{\begin{array}{cc}
O(s) & k \neq \bar{k}, l \neq \bar{l} \\
\varepsilon_{0}(s) & k=\bar{k}, l=\bar{l}
\end{array}\right\} \quad s \in(-\infty, t]
$$

where $\varepsilon_{0}(s)$ is arbitrary. Since by linearity, $\mathscr{F}_{i j k l}\left[\left.O(s)\right|_{-\infty} ^{t}\right]=0$, one obtains from equation (9) that for each choice of $\bar{k}, \bar{l}$, and $\varepsilon_{0}(s)$,

$$
\mathscr{F}_{i j k d}^{X}\left[\left.\varepsilon_{0}(s)\right|_{-\infty} ^{t}\right]=A_{m i} A_{n j} A_{p k} A_{q l} \mathscr{F}_{m n p q}^{X^{\prime}}\left[\left.\varepsilon_{0}(s)\right|_{-\infty} ^{t}\right] \text {, }
$$

which establishes the desired result.

Material symmetry restrictions can now be derived from equation (10) without using specific representations for the linear functionals. For example, assume that the material is isotropic. Then for each choice of $X^{\prime}$

$$
\mathscr{F}_{i j k k}^{X}\left[\left.\varepsilon_{0}(s)\right|_{-\infty} ^{t}\right]=\mathscr{F}_{i j k d}^{X}\left[\left.\varepsilon_{0}^{\prime}(s)\right|_{-\infty} ^{t}\right] \text {. }
$$

Combining equations (10) and (11), one obtains the condition

$$
\mathscr{F}_{i j k}^{X}\left[\left.\varepsilon_{0}(s)\right|_{-\infty} ^{t}\right]=A_{m i} A_{n j} A_{p k} A_{q l} \mathscr{F}_{\text {mnpq }}^{X}\left[\left.\varepsilon_{0}(s)\right|_{-\infty} ^{t}\right] \text {, }
$$


which must be satisfied for all choices of $A_{i j}$ satisfying equation (7). By a standard argument, this leads to a tensorial representation for $\mathscr{F}_{i j k l}^{X}$ in terms of two distinct linear scalar functionals and the Kronecker deltas,

$$
\mathscr{F}_{i j k k l}^{X}\left[\left.\varepsilon_{0}(s)\right|_{-\infty} ^{t}\right]=\lambda\left[\left.\varepsilon_{0}(s)\right|_{-\infty} ^{t}\right] \delta_{i j} \delta_{k l}+\mu\left[\left.\varepsilon_{0}(s)\right|_{-\infty} ^{t}\right]\left(\delta_{i k} \delta_{j l}+\delta_{i l} \delta_{j k}\right) \text {. }
$$

Equation (4) becomes, with an obvious redefinition of $\lambda$ and $\mu$,

$$
\sigma_{i j}(t)=\frac{1}{3}\left[G_{2}\left[\left.\varepsilon_{k k}(s)\right|_{-\infty} ^{t}\right]-G_{1}\left[\left.\varepsilon_{k k}(s)\right|_{-\infty} ^{t}\right]\right] \delta_{i j}+G_{1}\left[\left.\varepsilon_{i j}(s)\right|_{-\infty} ^{t}\right] .
$$

$G_{1}$ and $G_{2}$ have the usual physical interpretation as linear functionals for the response in shear and isotropic stress, respectively.

A similar result is obtained by interchanging the roles of stress and strain. In this case, for an isotropic material, the constitutive equation has the form:

$$
\varepsilon_{i j}(t)=\frac{1}{3}\left[J_{2}\left[\left.\sigma_{k k}(s)\right|_{-\infty} ^{t}\right]-J_{1}\left[\left.\sigma_{k k}(s)\right|_{-\infty} ^{t}\right]\right]+J_{1}\left[\left.\sigma_{i j}(s)\right|_{-\infty} ^{t}\right] .
$$

\section{Bending of a beam by terminal moments}

Consider a cylindrical body of arbitrary cross-section. Let the $x_{1}$-axis pass through the centroids of the cross-sections, and the $x_{2}$-and $x_{3}$-axes lie along its principal directions. The beam is subjected to time dependent bending moments $M(t)$ at its ends about the $x_{3}$-axis. Assumptions regarding the deformation and stress distribution histories arise as natural extensions of the usual semi-inverse approach in the linear elastic version of the problem. At each instant, the plane cross-sections have rotated without warping about the $x_{3}$-axis and remain perpendicular to the curve through the centroids, i.e. the neutral axis. This leads to an $\varepsilon_{11}$ strain history of the form

$$
\varepsilon_{11}\left(x_{2}, s\right)=-x_{2} / \rho(s), \quad s \in(-\infty, t],
$$

where $\rho(s)$ is the radius of curvature history of the neutral axis. In conjunction with this strain history, the stress history assumption is

$$
\left.\begin{array}{l}
\sigma_{i j}\left(x_{2}, s\right)=O(s), \quad i, j \neq 1 \\
\sigma_{11}\left(x_{2}, s\right) \neq 0
\end{array}\right\} s \in(-\infty, t] .
$$

For the present example, it will be more convenient to use the constitutive equation in form (15). Dependence of the arguments of the linear functionals on $s$ will be suppressed for notational simplicity. Then, by equations (15), (16), and (17), the equation for $\varepsilon_{11}$ gives

$$
\frac{-x_{2}}{\rho(t)}=\frac{1}{3} J_{2}\left[\sigma_{11}\right]+\frac{2}{3} J_{1}\left[\sigma_{11}\right] .
$$

The equations for the remaining normal strain components give

$$
\varepsilon_{22}(t)=\varepsilon_{33}(t)=\frac{1}{3}\left[J_{2}\left[\sigma_{11}\right]-J_{1}\left[\sigma_{11}\right]\right] .
$$


The shear strain components vanish, which is consistent with the kinematic assumption that the sections remain normal to the neutral axis.

The right hand sides of equations (18) and (19) are linear functionals in $\sigma_{11}$. Denoting these by $F_{1}$ and $F_{2}$, respectively, equations (18) and (19) become

$$
\begin{aligned}
& \frac{-x_{2}}{\rho(t)}=F_{1}\left[\sigma_{11}\right], \\
& \varepsilon_{22}(t)=\varepsilon_{33}(t)=F_{2}\left[\sigma_{11}\right] .
\end{aligned}
$$

If it is now assumed that $F_{1}$ has an inverse, $F_{1}^{-1}$, equation (20) gives

$$
\sigma_{11}\left(x_{2}, t\right)=F_{1}^{-1}\left[\frac{-x_{2}}{\rho}\right]=-x_{2} F_{1}^{-1}\left[\left.\frac{1}{\rho}(s)\right|_{-\infty} ^{t}\right]=-x_{2} F_{1}^{-1}\left[\frac{1}{\rho}\right] .
$$

The last step is justified by the fact that $F_{1}^{-1}$ is a linear operator on the time dependence of $-x_{2} / \rho(s)$. Since $x_{2}$ is an independent spatial variable, it acts as a constant with respect to $F_{1}^{-1}$. This separation of the space and time dependence plays a significant role in that it permits the usual results to be derived without any further comment regarding $F_{1}^{-1}$. In particular, the resultant force and bending moment about the $x_{2}$-axis can be shown to vanish because of the definition of the coordinate system. On calculating the bending moment $M(t)$ about the $x_{3}$-axis at each time $t$, we obtain

$$
\begin{aligned}
M(t) & =-\int_{\mathrm{A}} x_{2} \sigma_{11}\left(x_{2}, t\right) d A=I F_{1}^{-1}\left[\frac{1}{\rho}\right], \\
I & =\int_{\mathrm{A}} x_{2}^{2} d A .
\end{aligned}
$$

From equations (22) and (23) follow the well known result

$$
\sigma_{11}\left(x_{2}, t\right)=\frac{-M(t) x_{2}}{I} .
$$

This shows that the stress distribution is independent of the properties of a particular linear material and depends only on the current value of the bending moment. Finally, upon substituting equation (24) into equation (19), one sees that the transverse strains vary linearly with $x_{2}$.

\section{Pressurization of a hollow cylinder}

Consider a long hollow circular cylindrical body which is subjected to uniform pressure histories on its inner and outer surfaces. With respect to the obvious cylindrical coordinate system, the pressure $P_{a}(t)$ is applied at the inner radius $r=a$ while pressure $P_{b}(t)$ is applied at the outer radius $r=b$. The usual assumptions of 
axial symmetry and plane strain are

$$
u_{z}=u_{\theta}=0, \quad u_{r}=u(r, s), \quad a \leq r \leq b, \quad s \in(-\infty, t] .
$$

From equation (14), one obtains that

$$
\sigma_{r \theta}(t)=\sigma_{r z}(t)=\sigma_{z \theta}(t)=0
$$

and

$$
\begin{aligned}
& \sigma_{r r}(t)=\frac{1}{3}\left[G_{2}[D u]-G_{1}[D u]\right]+G_{1}\left[\frac{d u}{d r}\right], \\
& \sigma_{\theta \theta}(t)=\frac{1}{3}\left[G_{2}[D u]-G_{1}[D u]\right]+G_{1}\left[\frac{u}{r}\right], \\
& \sigma_{z z}(t)=\frac{1}{3}\left[G_{2}[D u]-G_{1}[D u]\right],
\end{aligned}
$$

where $D$ denotes the differential operator defined by

$$
D u(r, s)=\frac{d u}{d r}(r, s)+\frac{u(r, s)}{r}, \quad a \leq r \leq b, \quad s \in(-\infty, t] .
$$

It is now assumed that the operation of spatial differentiation can be interchanged with the $G_{1}$ and $G_{2}$ operators (which is the case in linear elasticity and viscoelasticity). Then on imposing the stress equilibrium equation

$$
\frac{d \sigma_{r}}{d r}+\frac{\sigma_{r}-\sigma_{\theta}}{r}=0, \quad a<r<b, \quad s \in(-\infty, t],
$$

at each instant, one obtains that

$$
\frac{1}{3} G_{2}[\hat{D} u]+\frac{2}{3} G_{1}[\hat{D} u]=0,
$$

where $\hat{D}$ is a differential operator defined by

$$
\hat{D} u=\frac{d^{2} u}{d r^{2}}+\frac{1}{r} \frac{d u}{d r}+\frac{u}{r^{2}}, \quad a<r<b, \quad s \in(-\infty, t] .
$$

The left hand side of equation (29) is a linear functional in $\hat{D} u$. If it is assumed that the null space of this operator is the zero element (which is again the case for linear elasticity and viscoelasticity), then the displacement field satisfies

$$
\hat{D} u=0, \quad a<r<b, \quad s \in(-\infty, t] .
$$

The solution to equations (30) and (31) is given by

$$
u(r, s)=A(s) r+\frac{B(s)}{r} .
$$

Again notice the separation of the spatial and time dependence. Substitution from 
equation (32) into equation (26) gives

$$
\begin{aligned}
& \sigma_{r r}(t)=\frac{2}{3} G_{2}[A]+\frac{1}{3} G_{1}[A]-\frac{1}{r^{2}} G_{1}[B], \\
& \sigma_{\theta \theta}(t)=\frac{2}{3} G_{2}[A]+\frac{1}{3} G_{1}[A]+\frac{1}{r^{2}} G_{1}[B], \\
& \sigma_{z z}(t)=\frac{1}{3}\left[G_{2}[A]-G_{1}[A]\right] .
\end{aligned}
$$

On imposing the boundary conditions on $\sigma_{r r}$ at $r=a, b$, one obtains expressions for $\left[2 G_{2}[A]+G_{1}[A]\right] / 3$ and $G_{1}[B]$ in terms of $P_{a}(t)$ and $P_{b}(t)$. This leads to the well known expressions for $\sigma_{r r}, \sigma_{\theta \theta}$, and $\sigma_{z z}$ in which they are shown to depend only on the current values of the pressures and are independent of the material properties.

\section{Final comments}

The last two examples suggest that any problem in elasticity or viscoelasticity which leads to expressions for stress or strain in which spatial and time dependence are separated can be formulated and solved for a general linear material, which satisfy certain reasonable properties. The displacement formulation of the St. Venant torsion problem and the spherically symmetric problem also fall into this category. If composition operations are defined, it should also be possible to include the plane problem. In effect, this extends the correspondence theory relating solutions in linear elasticity and viscoelasticity to linear materials.

\section{References}

[1] Gurtin, M. E. and E. Sternberg, "On the Linear Theory of Viscoelasticity", Arch. Rational Mech. Anal. 11 (1962) pp. 291-356.

[2] Wineman, A. S. and D. C. Stouffer, "A Constitutive Equation for Linear, Aging EnvironmentalDependent Viscoelastic Materials", Acta Mechanica 13 (1972) pp. 31-53. 